\title{
Double-arc volumetric modulated therapy improves dose distribution compared to static gantry IMRT and 3D conformal radiotherapy for adjuvant therapy of gastric cancer
}

\author{
Tao Zhang ${ }^{\dagger}$, Zhi-Wen Liang ${ }^{\dagger}$, Jun Han, Jian-Ping Bi, Zhi-Yong Yang and Hong Ma*
}

\begin{abstract}
Background: The objective of this study was to compare the dose distributions of RapidArc (RA), static gantry intensity-modulated radiotherapy (IMRT), and three-dimensional conformal radiotherapy (3DCRT) as adjuvant radiotherapy modalities for the treatment of gastric cancer.
\end{abstract}

Methods: Fifteen patients with gastric cancer that underwent limited lymphadenectomy of perigastric lymph nodes were included in this study. Dosimetric values for a total dose of $45 \mathrm{~Gy}$ (1.8 Gy/day) were calculated for the RapidArc, IMRT, and 3DCRT modalities. The following parameters were compared: $D_{99 \%}, D_{1 \%}, V_{95 \%}, V_{107 \%}$, and conformity and homogeneity index values $(\mathrm{Cl}$ and $\mathrm{HI}$, respectively) for the planned target volume (PTV). Dose volume histogram $(\mathrm{DVH})$ and dose distribution of the organs at risk (OAR), as the maximal dose to the spinal cord, $V_{30}$ and $V_{40}$ of the small bowel, and $V_{20}, V_{30}$ of liver and kidney were also assessed respectively.

Results: RA, IMRT, and 3DCRT all achieved desirable PTV coverage. However, RA and IMRT significantly decreased $\mathrm{D}_{1 \%}$ and $\mathrm{V}_{107 \%}$, and provided better $\mathrm{Cl}$ and $\mathrm{HI}$ values compared with 3DCRT $(P<0.05)$. Moreover, RA also achieved a significantly lower maximum dose for the spinal cord, liver $V_{30}$, and kidney $V_{20}$ compared to IMRT and 3DCRT; while the mean dose for these three organ types did not differ for the RA, IMRT, and 3DCRT plans.

Conclusions: Both RA and IMRT achieved favorable PTV coverage compared to 3DCRT. In addition, RA achieved better dosimetry than IMRT and 3DCRT, and provided better protection for the spinal cord, liver, and kidneys.

Keywords: Double-arc volumetric modulated arc therapy, Intensity-modulated radiotherapy, Three-dimensional conformal radiotherapy, Gastric cancer

\section{Background}

The INT0116 study revealed the survival benefits of postoperative radiotherapy for gastric cancer patients $[1,2]$. Moreover, both the 3-year and 11-year follow-up results affirmed the overall survival and disease-free survival benefits of radiotherapy [1, 2]. Despite these results, however, gastric cancer radiotherapy remains controversial. Specifically, concerns remain regarding radiation-induced toxicity. The toxicity levels reported

\footnotetext{
* Correspondence: wudajianzhu2004@163.com

${ }^{\dagger}$ Equal contributors

Cancer Center of Union Hospital, Tongji Medical College, Huazhong

University of Science and Technology, No. 13 Hangkong Road, Wuhan, Hubei 430022, China
}

(C) 2015 Zhang et al.; licensee BioMed Central. This is an Open Access article distributed under the terms of the Creative Commons Attribution License (http://creativecommons.org/licenses/by/4.0), which permits unrestricted use, distribution, and reproduction in any medium, provided the original work is properly credited. The Creative Commons Public Domain Dedication waiver (http://creativecommons.org/publicdomain/zero/1.0/) applies to the data made available in this article, unless otherwise stated.

for the INT0116 study included grade 3 (40\%), grade 4 (32\%), and gastrointestinal toxicity (33\%), and three cases involved treatment-related deaths. Consequently, treatment-related toxicity remains a limiting factor for the application of gastric cancer radiotherapy [1].

In recent years, three-dimensional conformal radiotherapy (3DCRT) and intensity modulated radiotherapy (IMRT) have been widely used for the treatment of cancer. These techniques address the drawbacks of conventional anteroposterior-posteroanterior techniques, such as under-dosage of target regions and excessive radiation to surrounding normal structures. An advantage of IMRT technology over 3DCRT for the treatment of nasopharyngeal carcinoma, prostate cancer, and lung 
cancer has been improved dose distribution within the target area, better dose hotspot control, and reduced radiation exposure to organs at risk (OAR), including the brain stem and spinal cord [3-5]. However, it continues to be debated whether IMRT or 3DCRT is better for gastric cancer radiotherapy $[6,7]$. In our previous study, IMRT was found to provide better target uniformity and conformality than four-field 3DCRT. However, IMRT did not reduce the dose applied to the OAR (e.g., the liver and kidneys) [8]. Therefore, the availability of new technologies is of great interest.

RapidArc (RA) is a type of dynamic IMRT that involves application of a rotation beam according to Otto's rotation theory of intensity-modulated radiation therapy. Briefly, by dynamically changing the gantry rotation speed, the shape of the multi collimator leaves, and the dose rate, RA can rapidly and efficiently achieve superior radiation dose distribution [9]. As such, RA technology has the potential to shorten treatment time and reduce the possibility of target movement during treatment, thereby increasing treatment accuracy. Currently, the literature available regarding RA mainly focuses on the treatment of breast, prostate, and lung cancer [10-12]. In contrast, only a few studies have reported clinical applications of RA for gastric cancer [13].

To date, neither 3D-CRT nor IMRT have shown a clear advantage in gastric cancer radiotherapy. This is mostly attributed to the extensive region of the OAR that is involved. It also remains to be determined whether RA technology would improve the outcome of gastric cancer radiotherapy. Therefore, the goal of this study was to compare the dose distribution of RA, static gantry IMRT, and 3DCRT for the radiotherapy treatment of gastric cancer using dosimetric analysis, and to evaluate which external radiation technology is best for the postoperative treatment of gastric cancer.

\section{Methods \\ Patients}

Between October 2010 and December 2011, 15 gastric cancer patients who underwent D1 surgery at our hospital were enrolled in this study. According to the 2010 AJCC staging manual for gastric cancer [14], there were 6 T3 stage patients and $9 \mathrm{~T} 4$ stage patients. In addition, the lymph nodes in 7/9 patients were negative. The primary tumors were located in the cardia $(n=4)$, the pylorus $(n=6)$, or in the gastric body $(n=5)$. For this retrospective study, all of the patients completed 3D-CRT treatment before December 2011. Based on the CT images that were collected, three different treatment plans (3DCRT, IMRT, and RapidArc) were generated in order to compare the dose distributions of each. This study was approved by the ethics committee of the hospital and informed consent was obtained from all of the patients.

\section{Patient positioning}

Each patient achieved a supine position with their hands over their chest. This position was then fixed using a thermoplastic mask. Patients fasted $4 \mathrm{~h}$ before the simulation computed tomography $(\mathrm{CT})$ scans, and they were administered iohexol in $200 \mathrm{ml}$ water orally $10 \mathrm{~min}$ before positioning. Enhanced CT scans were performed with a slice thickness of $3 \mathrm{~mm}$. CT images were transferred to the Aria Network (Varian system) and were reconstructed using the ECLIPSE treatment planning system (Version 11, Varian Medical System, Palo Alto, CA, USA).

\section{Target and OAR delineation}

According to Report 62 [15] of the International Commission on Radiation Units and Measurements that refers to $\mathrm{CT}$ and other imaging methods, clinical target volume (CTV) included the anastomosis, tumor bed, and regional lymph nodes. The planning target volume (PTV) was defined as a uniform $5 \mathrm{~mm}$ expansion of the CTV. The liver, left kidney, right kidney, spinal cord, small intestine, heart, and other OAR were delineated step-by-step as previously described [16].

\section{Treatment planning}

Three radiotherapy treatment plans were generated using the Varian Eclipse treatment planning system (Version 11, Varian Medical System, Palo Alto, CA, USA) by an experienced physicist. For each of the plans, $6 \mathrm{MV}$ photon beams from a Trilogy machine (Varian Medical System) were used and dose calculations were performed using the Acrous XB algorithm. For 4-field 3DCRT, the center of the PTV was designated the center of the irradiation field. The box technique in 3DCRT was found to better protect the OAR compared with half beam techniques and the use of wedges. Therefore, in this study, the box technique was selected and the incident angles used were $0^{\circ}, 90^{\circ}, 180^{\circ}$, and $270^{\circ}$. The dose applied at the center of the central plane was also set as the reference. For fixed-field sliding window IMRT, the gantry angle was fixed at $0^{\circ}, 35^{\circ}, 90^{\circ}, 180^{\circ}$, and $315^{\circ}$. For $\mathrm{RA}$, the coplanar double arc included $358^{\circ}$ of rotation therapy, with $179^{\circ}$ as the starting angle and $330^{\circ}$ as the end angle. A maximum dose rate of 600 monitor units $(\mathrm{MU}) / \mathrm{min}$ was applied. For all three plans, the prescribed dose for the PTV was $45 \mathrm{~Gy} / 25 \mathrm{~F}$. This dose was established to ensure that $>95 \%$ of the PTV received $45 \mathrm{~Gy}$, and $99 \%$ of the PTV received $>42.75 \mathrm{~Gy}$. For the OAR, less than $30 \%$ of the whole liver volume was allowed to receive $>30 \mathrm{~Gy}\left(\mathrm{~V}_{30} \leq 30 \%\right)$. For the contralateral kidney, the volume exposed to more than $20 \mathrm{~Gy}$ was also limited to $<30 \%\left(\mathrm{~V}_{20}<30 \%\right)$. The allowed mean dose $\left(D_{\text {mean }}\right)$ for each kidney was $<18.0 \mathrm{~Gy}$, and the maximum allowed dose for the spinal cord was $<45$ Gy. 
Radiation exposure to the small intestine was also minimized during the generation of the radiotherapy treatment plans. The $\mathrm{V}_{40}$ and $\mathrm{V}_{25}$ for the heart were $<30 \%$ and $<50 \%$, respectively $[16,17]$.

\section{Evaluation and comparison of the three treatment plans} Dose distributions to the target organs and the OAR for the fifteen patients were assessed. Dose volume histograms (DVH) were also generated and compared, with the specific dosimetry parameters evaluated as follows:

To evaluate target coverage, the dose received by $99 \%$ and $1 \%$ of the volume (e.g., $\mathrm{D}_{99 \%}$ and $\mathrm{D}_{1 \%}$, respectively) were defined as metrics for the minimal and maximal doses [18]. The volumes receiving at least $95 \%$ and $107 \%$ of the prescribed dose $\left(\mathrm{V}_{95 \%}\right.$ and $\mathrm{V}_{107 \%}$, respectively), as well as target homogeneity and conformal index values ( $\mathrm{HI}$ and $\mathrm{CI}$, respectively), were also compared. HI was calculated as: $\mathrm{HI}=(\mathrm{D} 2-\mathrm{D} 98) / \mathrm{D} 50$. The greater the $\mathrm{HI}$ value, the poorer the uniformity of the dose distribution [19]. CI was calculated as follows: $\mathrm{V}_{\mathrm{T}}$, ref $/ \mathrm{V}_{\mathrm{T}} \times \mathrm{V}_{\mathrm{T}}$, ref $/ \mathrm{V}_{\text {ref }}$, where $\mathrm{V}_{\mathrm{T}, \text { ref }}$ is the volume of target covered by the reference isodose line, $\mathrm{V}_{\mathrm{T}}$ is the target volume (= PTV), and $\mathrm{V}_{\text {ref }}$ is the volume of tissue covered by the reference isodose line. The value of CI varies between 0 and 1 , and a value closer to one indicates better conformity of dose to the PTV [20, 21]. The equivalent uniform dose (EUD) for each PTV was compared.

Dose distribution to vital organs, including kidneys, liver, small intestine, and spinal cord, were also assessed. The parameters that were compared included mean dose $\left(D_{\text {mean }}\right)$ and $V_{20}$ for the kidneys $\left(V_{20}\right.$ is the percentage volume of the kidneys that received at least $20 \mathrm{~Gy}$ ), $D_{\text {mean }}$ and $V_{30}$ for liver, $V_{30}$ and $V_{40}$ for small intestine, and maximum dose $\left(D_{\max }\right)$ and $\mathrm{D}_{1 \%}$ for the spinal cord. Monitor units (MU) were also compared between three plans.

\section{Statistical analysis}

SPSS (version 17.0, SPSS Inc., Chicago, IL, USA) was used for data analysis. Non-parametric Wilcoxon tests or two-tailed $t$-tests were performed to compare groups. A $p$-value less than 0.05 was considered statistically significant.

\section{Results}

\section{PTV comparison}

All three plans fulfilled the dose requirement, and there were no significant differences in the $\mathrm{D}_{99 \%}$ minimum dose and the $\mathrm{V}_{95 \%}$ target volume between them. However, the RA plan did significantly reduce the maximum target dose and the high dose volume (e.g., $\mathrm{D}_{1 \%}$ and $\mathrm{V}_{107 \%}$, respectively) compared to the 3DCRT and IMRT plans, and the difference between the 3DCRT and IMRT plans was not significant. Both IMRT and RA significantly lowered the EUD of the PTV compared to 3DCRT $(P<0.05)$. Regarding target uniformity, both IMRT and RA also improved the PTV uniformity compared to 3DCRT $(P<0.05)$. In addition, the $\mathrm{CI}$ values were $0.91 \pm$ 0.02 for RA, $0.89 \pm 0.04$ for IMRT, and $0.71 \pm 0.01$ for 3DCRT. The former was significantly closer to a value of 1 compared with the IMRT and 3DCRT plans $(P<0.05)$ (Tables 1 and 2; Figs. 1 and 2).

\section{Evaluation of OAR}

Previous research has shown that the $D_{\text {mean }}$ and $V_{30}$ for the liver are important predictors of radiation-induced liver damage [8]. In the present study, $\mathrm{V}_{30}$ for the liver was $(12.32 \pm 1.61) \%$ for 3DCRT, $(12.73 \pm 1.33) \%$ for IMRT, and $(6.90 \pm 1.41) \%$ for RA. The latter was significantly lower than the other two methods $(P<0.05)$. In addition, the $D_{\text {mean }}$ for the liver was $17.61 \pm 0.82$ Gy for 3DCRT, $14.22 \pm 0.23$ Gy for IMRT, and $15.31 \pm 1.11$ Gy for RA. Both IMRT and RA reduced the average radiation dose for the liver, yet the difference was not significant (Tables 1 and 2; Fig. 2). In a previous study by Matzinger and Dawson [16, 17], the recommended tolerance doses for the kidneys were $\mathrm{V}_{20}<30 \%$ and $D_{\text {mean }}<18$ Gy. In the present study, the $V_{20}$ for the left or right kidney with the RA plan was lower than that for the IMRT and 3DCRT plans. Specifically, RA treatment decreased the $\mathrm{V}_{20}$ of the left kidney by $25.17 \%$, and the right kidney by $33.94 \%$. In addition, the average dose for both kidneys was higher for both the IMRT and RA plans compared to 3DCRT, although the difference was not significant $(P>0.05)$ (Tables 1 and 2; Fig. 2).

The $V_{30}$ and $V_{40}$, as well as the $D_{\text {mean }}$, for the small intestine were also assessed. Compared with 3DCRT, IMRT and RA only moderately reduced $V_{30}$ and $V_{40}$, and the differences were not significant. In contrast, RA slightly increased the $D_{\text {mean }}$ of the small intestine, although this difference was also not significant $(P>0.05)$ (Tables 1 and 2; Fig. 2).

For the spinal cord, all three plans fulfilled the dose requirements. The maximum radiation doses for $\mathrm{D}_{1 \%}$ were $32.98 \pm 0.74$ Gy for 3DCRT, $31.01 \pm 0.29$ Gy for IMRT, and $27.80 \pm 0.75$ Gy for RA. Compared with 3DCRT, RA significantly lowered the spinal cord $D_{\max }$ value by $15.71 \%$ (Tables 1 and 2; Fig. 2).

\section{Comparison of MU and delivery parameters}

The MU for IMRT and RA were $694.25 \pm 3.91$ and $399.00 \pm 6.81$, respectively. Thus, RA significantly reduced the radiation dose received by $42.5 \%$ compared with IMRT. However, RA required a greater number of MU than 3DCRT. In addition, a physical wedge was not used for the 3DCRT treatments since this could increase 
Table 1 Summary of the DVH parameters examined

\begin{tabular}{|c|c|c|c|c|}
\hline \multicolumn{2}{|l|}{ Parameters } & \multirow{2}{*}{$\frac{\text { 3DCRT Mean } \pm \text { SD }}{49.9 \pm 0.29}$} & \multirow{2}{*}{$\frac{\text { IMRT Mean } \pm \text { SD }}{48.2 \pm 0.17}$} & \multirow{2}{*}{$\frac{\text { RA Mean } \pm \text { SC }}{48.4 \pm 0.24}$} \\
\hline PTV & $D_{1 \%}(G y)$ & & & \\
\hline & $D_{99 \%}(G y)$ & $40.5 \pm 0.43$ & $40.3 \pm 0.41$ & $40.1 \pm 0.37$ \\
\hline & $V_{95 \%}(\%)$ & $96.6 \pm 0.61$ & $98.9 \pm 0.33$ & $98.5 \pm 0.62$ \\
\hline & $V_{107 \%}(\%)$ & $13.6 \pm 3.7$ & $1.02 \pm 0.01$ & $1.02 \pm 0.01$ \\
\hline & EUD (Gy) & $47.3 \pm 0.09$ & $46.8 \pm 1.45$ & $46.4 \pm 0.06$ \\
\hline & $\mathrm{HI}$ & $0.1 \pm 0.01$ & $0.05 \pm 0.01$ & $0.07 \pm 0.01$ \\
\hline & $\mathrm{Cl}$ & $0.71 \pm 0.02$ & $0.89 \pm 0.04$ & $0.90 \pm 0.02$ \\
\hline \multirow[t]{2}{*}{ Normal liver } & $D_{\text {mean }}(G y)$ & $17.6 \pm 0.82$ & $14.2 \pm 0.73$ & $15.3 \pm 1.1$ \\
\hline & $V_{30}(\%)$ & $12.3 \pm 1.6$ & $12.7 \pm 1.3$ & $6.90 \pm 1.4$ \\
\hline \multirow[t]{2}{*}{ Left kidney } & $D_{\text {mean }}(G y)$ & $13.2 \pm 1.21$ & $15.3 \pm 0.63$ & $14.1 \pm 0.61$ \\
\hline & $V_{20}(\%)$ & $29.9 \pm 2.5$ & $27.7 \pm 1.8$ & $22.4 \pm 3.6$ \\
\hline \multirow[t]{2}{*}{ Right kidney } & $D_{\text {mean }}(G y)$ & $11.9 \pm 1.4$ & $13.5 \pm 0.65$ & $12.2 \pm 0.90$ \\
\hline & $V_{20}(\%)$ & $19.2 \pm 1.1$ & $16.2 \pm 1.1$ & $12.7 \pm 1.3$ \\
\hline \multirow[t]{3}{*}{ Small bowel } & $D_{\text {mean }}(G y)$ & $13.1 \pm 0.83$ & $12.4 \pm 0.39$ & $13.1 \pm 0.76$ \\
\hline & $V_{30}(\%)$ & $17.2 \pm 0.61$ & $16.5 \pm 0.67$ & $15.6 \pm 0.83$ \\
\hline & $V_{40}(\%)$ & $11.0 \pm 0.38$ & $9.78 \pm 0.93$ & $9.15 \pm 0.44$ \\
\hline Spinal cord & $\mathrm{D}_{1 \%}(\mathrm{~Gy})$ & $33.0 \pm 0.74$ & $31.0 \pm 0.29$ & $27.8 \pm 0.75$ \\
\hline$M U$ & & $250 \pm 3.4$ & $694 \pm 3.9$ & $399 \pm 6.8$ \\
\hline
\end{tabular}

Abbreviations: 3DCRT: 3D conformal radiation therapy; IMRT: intensity-modulated radiation therapy; RA: RapidArc; PTV: planned tumor volume; $\mathrm{D}_{\mathrm{n} \%}$ : dose received by $n \%$ of the volume; Gy: Gray (unit); $\mathrm{Vx} \%$ : the volume receiving $\geq \mathrm{x} \%$ of the prescribed dose; EUD: equivalent uniform dose; HI: homogeneity index; Cl: conformity index; $D_{\text {mean }}$ : the mean dose for the organ; $V_{n}$ : the volume receiving $n$ dose of radiation (Gy); MU: monitor units; EUD: equivalent uniform dose

Table 2 Differences among the three methods with regard to the DVH parameters

\begin{tabular}{|c|c|c|c|c|c|}
\hline \multirow[t]{2}{*}{ Parameters } & & \multicolumn{4}{|l|}{$P$-values } \\
\hline & & Overall & 3DCRT vs. IMRT & 3DCRT vs. RA & IMRT vs. RA \\
\hline \multirow[t]{7}{*}{$\overline{\text { PTV }}$} & $D_{1 \%}(G y)$ & 0.003 & $3 \mathrm{DCRT}>\mathrm{IMRT}^{* *}$ & $3 \mathrm{DCRT}>\mathrm{RA}^{* *}$ & - \\
\hline & $\mathrm{D}_{99 \%}(\mathrm{~Gy})$ & 0.803 & - & - & - \\
\hline & $V_{95 \%}(\%)$ & 0.533 & - & - & - \\
\hline & $V_{107 \%}(\%)$ & 0.005 & 3DCRT > IMRT* & $3 \mathrm{DCRT}>\mathrm{RA} A^{*}$ & - \\
\hline & EUD (Gy) & 0.012 & $3 \mathrm{DCRT}>\mathrm{IMRT}^{*}$ & $3 \mathrm{DCRT}>\mathrm{RA} A^{*}$ & - \\
\hline & $\mathrm{HI}$ & 0.03 & $3 \mathrm{DCRT}>\mathrm{IMRT} *$ & $3 \mathrm{DCRT}>\mathrm{RA}^{*}$ & - \\
\hline & $\mathrm{Cl}$ & 0.001 & $3 \mathrm{DCRT}<\mathrm{IMRT} *$ & $3 \mathrm{DCRT}<\mathrm{RA}^{*}$ & - \\
\hline \multirow[t]{2}{*}{ Normal liver } & $D_{\text {mean }}(G y)$ & 0.058 & $3 \mathrm{DCRT}>\mathrm{IMRT} \mathrm{T}^{* *}$ & - & - \\
\hline & $V_{30}(\%)$ & 0.006 & - & $3 \mathrm{DCRT}>\mathrm{RA}^{* *}$ & $I M R T>R A^{* *}$ \\
\hline \multirow[t]{2}{*}{ Left kidney } & $D_{\text {mean }}(G y)$ & 0.335 & - & - & - \\
\hline & $V_{20}(\%)$ & 0.137 & - & $3 \mathrm{DCRT}>\mathrm{RA}^{*}$ & - \\
\hline \multirow[t]{2}{*}{ Right kidney } & $D_{\text {mean }}(G y)$ & 0.912 & - & - & - \\
\hline & $V_{20}(\%)$ & 0.005 & - & $3 \mathrm{DCRT}>\mathrm{RA}^{* *}$ & - \\
\hline \multirow[t]{3}{*}{ Small bowel } & $D_{\text {mean }}(G y)$ & 0.657 & - & - & - \\
\hline & $V_{30}(\%)$ & 0.075 & - & - & - \\
\hline & $V_{40}(\%)$ & 0.453 & - & - & - \\
\hline Spinal cord & $D_{1 \%}(G y)$ & 0.011 & - & $3 \mathrm{DCRT}>\mathrm{RA}^{*}$ & $I M R T>R A^{*}$ \\
\hline $\mathrm{MU}$ & & 0.001 & IMRT $>3$ DCRT $T^{* *}$ & & $I M R T>R A^{* *}$ \\
\hline
\end{tabular}

Abbreviations: 3DCRT: 3D conformal radiation therapy; IMRT: intensity-modulated radiation therapy; RA: RapidArc; PTV: planned tumor volume; $\mathrm{D}_{\mathrm{n} \%}$ : dose received by $n \%$ of the volume; Gy: Gray (unit); $\mathrm{Vx} \%$ : the volume receiving $\geq \mathrm{x} \%$ of the prescribed dose; EUD: equivalent uniform dose; HI: homogeneity index; Cl: conformity index; $D_{\text {mean }}$ : the mean dose for the organ; $V_{n}$ : the volume receiving $n$ dose of radiation (Gy); MU: monitor units ${ }^{*} P<0.05 ;{ }^{* *} P<0.01$ 


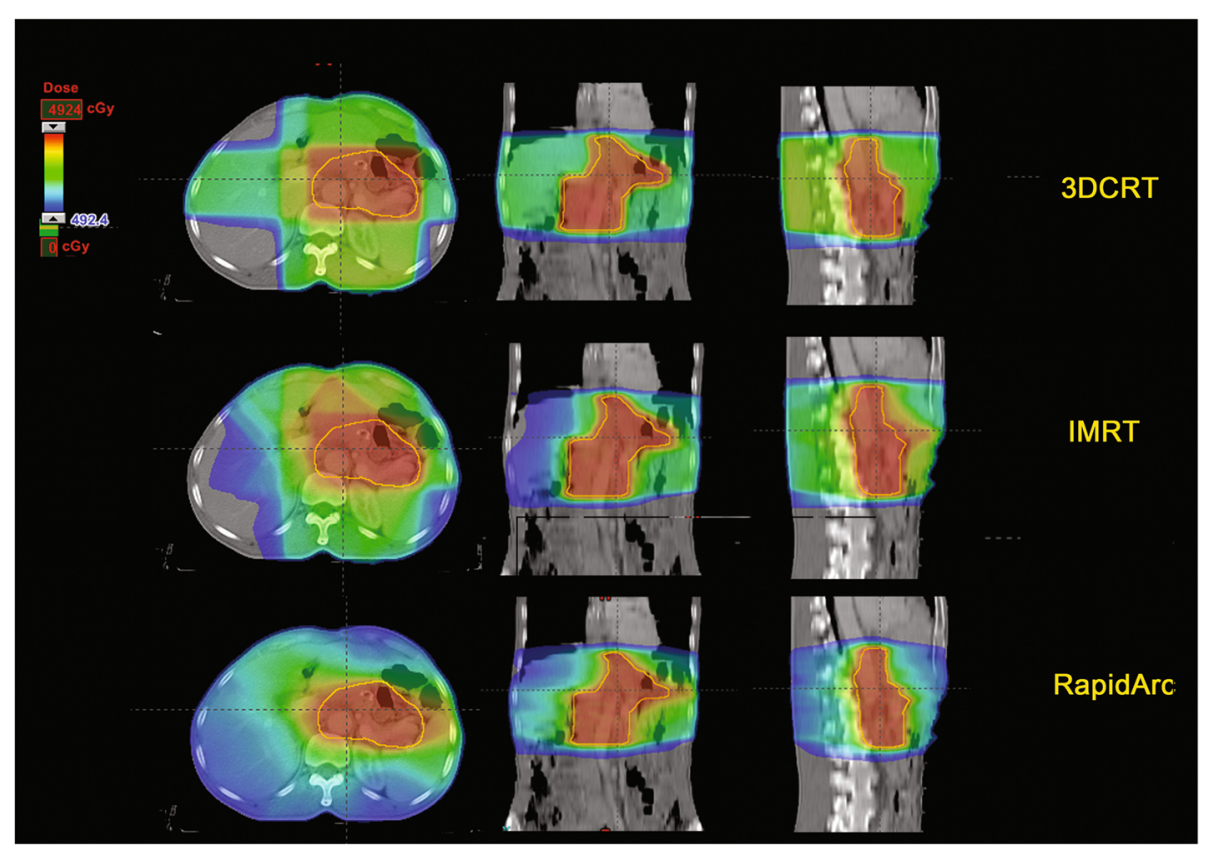

Fig. 1 Comparison of the PTV isodose distributions achieved with 3DCRT, IMRT, and RapidArc adjuvant radiotherapy modalities
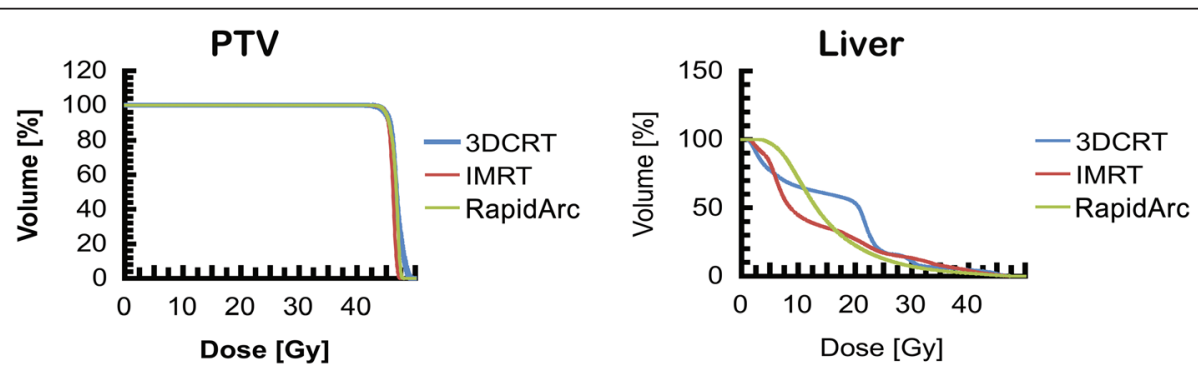

L Kidney
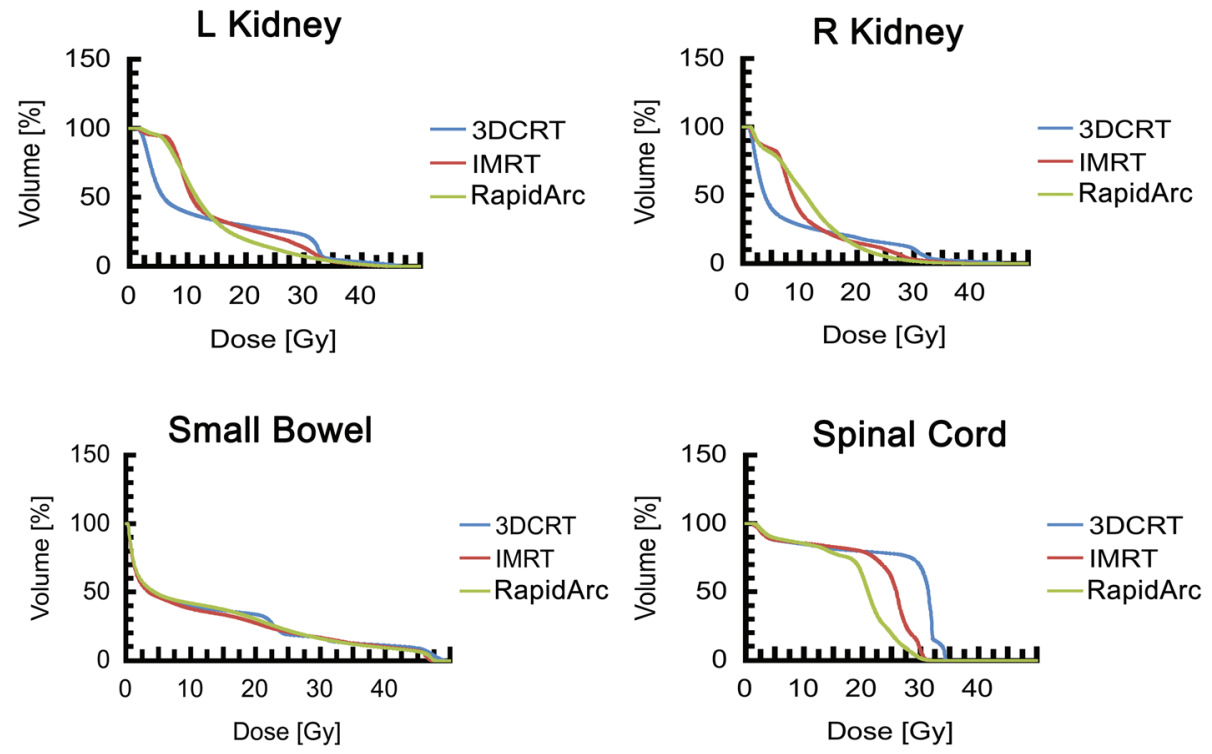

Fig. 2 Mean dose volume histograms for PTV, CTV, OAR, and healthy tissue for global analysis according to treatment plan. 3DCRT: 3D conformal radiation therapy (blue); IMRT: intensity-modulated radiation therapy (red); RapidArc: double-arc RapidArc (green) 
the number of MU, and it could also increase the potential for leakage radiation (Tables 1 and 2). The dose rate for each technique was $400 \mathrm{MU} / \mathrm{min}$ for 3DCRT, 600 $\mathrm{MU} / \mathrm{min}$ for IMRT, and nearly $600 \mathrm{MU} / \mathrm{min}$ for ARC. Thus, the relative treatment times for each technique were: $3.2 \pm 0.3 \mathrm{~min}$ for RA, $6.6 \pm 1.2 \mathrm{~min}$ for IM, and $4.2 \pm 0.5 \mathrm{~min}$ for CRT.

\section{Discussion}

Currently, postoperative chemoradiation is one of the main treatments for cases of gastric cancer with poor prognosis. However, due to the proximity of this region to many vital organs, it remains a challenge to effectively cover the target area and protect neighboring vital organs. For adjuvant radiotherapy modalities, it has been difficult to achieve an ideal dose distribution with traditional 3DCRT, while IMRT is able to simultaneously optimize target dose and decrease exposure of OAR. IMRT has also been shown to effectively improve local tumor control, to reduce the extent of radiation damage to normal tissues, and to improve patient quality of life [22]. However, in our previous study of 3DCRT and IMRT for the treatment of gastric cancer, radiation dosimetry data indicated that IMRT did not show a significant advantage over 3DCRT, with 3DCRT being superior to IMRT for $\mathrm{V}_{20}$ of the left and right kidneys [8]. Therefore, new radiotherapy techniques for the treatment of gastric cancer are still needed.

RA technology has the potential to shorten treatment time and reduce the possibility of target movement during treatment, which would serve to increase treatment accuracy [18]. RA has previously been applied to the treatment of many types of tumors [23, 24]. For example, in work by Verbakel et al. [25], twelve patients with advanced head and neck cancer received IMRT versus RA radiation therapy. Treatment with RA was found to improve target dose uniformity and to reduce exposure of neighboring OAR. Furthermore, double arc RA provided additional dosimetric advantages compared to single arc RA and IMRT. These advantages were confirmed with the treatment of lung cancer and prostate cancer with double arc RA $[11,12]$. However, for gastric cancer radiation therapy, the shape of the radiation target is irregular and the surrounding organs, including the liver and kidneys, have a low tolerance for radiation. Thus, it remains to be determined whether a rotary volumetric IMRT technique will be advantageous for gastric cancer radiotherapy.

The cohort studied included 15 postoperative gastric cancer patients. Based on the location of their lesions and CT imaging, 3DCRT (4-field), IMRT (5-field), or RA treatment plans were applied. The prescription dose included $45 \mathrm{~Gy} / 25 \mathrm{~F}$ applied to the PTV, with $>95 \%$ of the PTV receiving 45 Gy and $99 \%$ of the PTV receiving
42.75 Gy. All three plans met the dose requirements and there were no significant differences between them. Furthermore, IMRT and RA reduced the target maximum dose and the high dose range $\left(D_{1 \%}, V_{107 \%}\right)$ compared to 3DCRT. IMRT and RA were also superior to 3DCRT for target volume uniformity. The CI value for RA was significantly closer to one than the $\mathrm{CI}$ values for IMRT and 3DCRT, suggesting an improved conformality was achieved. For targets with larger and more complex shapes, RA was found to provide better dose distribution, better PTV target conformality, and better target dose distribution, and these results are consistent with previous studies [13]. Thus, RA has the potential to reduce treatment-related side effects.

In the early studies of organ tolerance to ionizing radiation, radiosensitivity of the liver may have been underestimated. Tolerance doses were limited according to the risk of RT-induced liver disease, and the mean dose and V30 for the liver were considered important dosimetric parameters associated with increased toxicity risk [26]. Meanwhile, more recent studies have shown that normal liver cells are sensitive to radiation, especially when the liver is infected with hepatitis B virus [26]. Accordingly, Dawson et al. [27] have suggested that the tolerance dose for the liver should be less than $30 \%$ for $V_{30}$, and the $D_{\text {mean }}$ should be less than 30 Gy. For cases involving hepatitis $B$ infection, the $D_{\text {mean }}$ should be less than 23 Gy. Furthermore, according to the Quantitative Analyses of Normal Tissue Effects in the Clinic (QUANTEC) effort, the mean liver dose should be less than 28 Gy in 2-Gy fractions for primary liver cancer, and should be less than 32 Gy in 2-Gy fractions for liver metastases [26]. In the present study, liver $\mathrm{V}_{30}$ was $(12.32 \pm 1.61) \%$ for 3DCRT, $(12.73 \pm 1.33) \%$ for IMRT, and $(6.90 \pm 1.41) \%$ for RA, with the latter being significantly lower than the two former values $(P<0.05)$. Liver $D_{\text {mean }}$ was $17.61 \pm 0.82 \mathrm{~Gy}$ for 3DCRT, $14.22 \pm 0.23 \mathrm{~Gy}$ for IMRT, and $15.31 \pm 1.11$ Gy for RA, and these did not significantly differ. Compared with 3DCRT and IMRT, RA significantly reduced liver $V_{30}$, yet did not affect the average liver dose. Furthermore, despite the significant reduction in liver $\mathrm{V}_{30}$, an analysis of volume from the DVH showed that $V_{10}$ increased. These results are consistent with those reported for a liver cancer radiation treatment study performed by Kuo et al. [28].

The kidney is another important organ that is threatened by gastric cancer radiotherapy. Kidney tissue is radiosensitive, and the recommended radiation tolerance doses are 23 Gy for the whole kidney, 30 Gy for $2 / 3$ of the kidney, and 50 Gy for $1 / 3$ of the kidney. A study by Jansen et al. further suggested that the average renal dose was less important than $V_{20}$. Therefore, it is recommended that $<70 \%$ of the kidney volume should receive 20 Gy $\left(V_{20}<70 \%\right)$, while the $V_{20}$ for the contralateral 
kidney should be $<30 \%$ [29]. In total, kidney tissues exposed to more than 20 Gy should not exceed $50 \%$ of the whole kidney, otherwise, radiation-induced damage to the kidney may occur, such as a decrease in the glomerular filtration rate and/or renal failure. Thus, an ongoing goal is to reduce the radiation dose to kidneys during postoperative radiotherapy for gastric cancer. Minn et al. [30] studied the dosimetry, efficacy, and toxicity of radiotherapy planning with 3DCRT and IMRT for 57 cases of gastric cancer, and IMRT was found to reduce kidney $\mathrm{V}_{20}$. In our previous study, no obvious difference in the $\mathrm{V}_{20}$ of kidney between IMRT and 3DCRT was observed, although IMRT exhibited favorable tumor coverage and superiority in protecting the spinal cord and liver. However, this superiority was not observed in the kidney compared with 3D-CRT. Thus, IMRT does not appear to represent a superior treatment for gastric cancer [8]. Similarly, in our subsequent single arc RA study, kidney radiation dose was not significantly reduced, yet double arc RA significantly decreased kidney $\mathrm{V}_{20}$ compared to IMRT and 3DCRT for both kidneys. Meanwhile, there was no obvious difference in the $\mathrm{D}_{\text {mean }}$ for both kidneys among the 3D-CRT, IMRT, and RA treatments. Taken together, these results suggest that RA can provide a protective effect for kidneys compared to IMRT.

Gastrointestinal toxicity is the main limiting factor for the application of radiation therapy to gastric cancer. Correspondingly, the key to reducing toxicity due to radiotherapy is to control the exposure of the gastrointestinal tract to radiation. In many studies, IMRT and RA have been shown to reduce the radiation dose to the gastrointestinal tract during abdominal radiation therapy. For example, Minn et al. [30] demonstrated that IMRT reduced intestinal $V_{45}$ compared to 3DCRT. In another study of 14 cases of abdominal metastases treated with radiation therapy, Mario et al. [30] reported that RA and IMRT reduced the average dose and maximum dose to the stomach and small intestine compared to 3DCRT. However, the difference was not significant. In the present study, the average dose $\left(D_{\text {mean }}\right)$ for the small intestine, as well as $V_{30}$ and $V_{40}$, were examined. $D_{\text {mean }}$ for the small intestine did not significantly differ among the three planning methods, yet a DVH chart analysis showed that IMRT and RA increased $V_{10}$ and reduced $\mathrm{V}_{30}$ and $\mathrm{V}_{40}$ compared to 3DCRT. Thus, the volume of the low dose region increased concomitant with a decrease in the volume of the high dose region. These results are consistent with the observation that the average dose did not show a significant difference.

The spinal cord is a long, thin, tubular bundle of nervous tissue and it is susceptible to injury from local high doses of radiation. Kirkpatrick et al. [31] reported that the incidence rate for radiation myelitis is $0.2,6$, and
$50 \%$ the total dose for $50 \mathrm{~Gy}, 60 \mathrm{~Gy}$, and_69 Gy, respectively, when administered at the conventional fraction of 2-Gy per day. In addition, according to the Radiation Oncology Group of the European Organisation for Research and Treatment of Cancer, the maximal radiation dose that should be applied to the spinal cord is $45 \mathrm{~Gy}$, and it should not exceed $40 \mathrm{~Gy}$ if oxaliplatin chemotherapy is administered as well [16]. Therefore, the maximum dose for the spinal cord is generally set at no more than $45 \mathrm{~Gy}$. In the present study, the doses applied to the spinal cord with each of the three techniques were all within the tolerated dose. Moreover, compared to 3DCRT, the $\mathrm{D}_{\max }$ for the spinal cord with RA was significantly reduced by up to $15.71 \%$.

RA is an adjuvant radiotherapy modality that has recently been developed and has been used to deliver high doses of radiation to a variety of tumors. However, its role in the treatment of gastric cancer remains controversial due to the irregular target volumes involved and the low radiation tolerance of surrounding critical organs. In our previous study, RA provided superior dose homogeneity compared with 3DCRT and IMRT, but not better protection of the OAR. Moreover, while the single arc technique was unsuccessful, the double arc technique was able to achieve the same dose distribution as IMRT, while significantly sparing the OAR and proximal healthy tissue. This improved protection of liver and kidney tissues compared with IMRT suggests a higher dose could be applied to a target volume using double arc RA. However, it is important to consider the limitations of our study as well. First, a respiratory gating technique was not used, and its influence on the dose distribution was not investigated. In addition, the present study had a small sample size and did not evaluate clinical efficacy and toxicity. Therefore, further studies are needed to confirm the technical feasibility of applying double arc RA to the treatment of gastric cancer, and these should include a larger sample size and evaluations of clinical efficacy and toxicity.

\section{Conclusions}

In summary, double arc RA reduced the maximum dose applied to the target area, it improved the conformality and uniformity of radiation, and it provided sufficient PTV coverage. Moreover, compared to 3DCRT and IMRT, double arc RA significantly reduced liver $\mathrm{V}_{30}$, kidney $V_{20}$, and the spinal cord maximum dose. Furthermore, RA was found to provide the best protection from high doses of radiation. Therefore, in this comparative study, a theoretical foundation for the clinical application of radiation for gastric cancer is demonstrated. Despite the small sample size, the results are very promising and further studies are needed to confirm these results. 


\section{Abbreviations}

IMRT: Intensity-modulated radiotherapy; 3DCRT: Three-dimensional conformal radiotherapy; PTV: Planned target volume; DVH: Dose volume histogram; OAR: Organs at risk; RA: RapidArc; CT: Computed tomography; CTV: Clinical target volume.

\section{Competing interests}

The authors declare that they have no competing interests.

\section{Authors' contributions}

TZ and Z-WL contributed significantly to the design and concept of this study, HM drafted the manuscript. JH, J-PB, and Z-YY contributed to optimization of the treatment plans. All of the authors have read and approved the final manuscript.

\section{Received: 12 December 2014 Accepted: 13 May 2015}

\section{Published online: 19 May 2015}

\section{References}

1. Macdonald JS, Smalley SR, Benedetti J, Hundahl SA, Estes NC, Stemmermann GN, et al. Chemoradiotherapy after surgery compared with surgery alone for adenocarcinoma of the stomach or gastroesophageal junction. N Engl J Med. 2001;345(10):725-30.

2. Smalley SR, Benedetti JK, Haller DG, Hundahl SA, Estes NC, Ajani JA, et al. Updated analysis of SWOG-directed intergroup study 0116: a phase III trial of adjuvant radiochemotherapy versus observation after curative gastric cancer resection. J Clin Oncol. 2012;30(19):2327-33.

3. Kam MK, Chau RM, Suen J, Choi PH, Teo PM. Intensity-modulated radiotherapy in nasopharyngeal carcinoma: dosimetric advantage over conventional plans and feasibility of dose escalation. Int J Radiat Onco Biol Phys. 2003;56(1):145-57.

4. Hummel S, Simpson EL, Hemingway P, Stevenson MD, Rees A. Intensitymodulated radiotherapy for the treatment of prostate cancer: a systematic review and economic evaluation. Health Technol Assess. 2010;14(47):1-108. iii-iv.

5. Murshed H, Liu HH, Liao Z, Barker JL, Wang X, Tucker SL, et al. Dose and volume reduction for normal lung using intensity-modulated radiotherapy for advanced-stage non-small-cell lung cancer. Int J Radiat Oncol Biol Phys. 2004;58(4):1258-67.

6. Milano MT, Garofalo MC, Chmura SJ, Farrey K, Rash C, Heimann R, et al. Intensity-modulated radiation therapy in the treatment of gastric cancer: early clinical outcome and dosimetric comparison with conventional techniques. Br J Radiol. 2006;79(942):497-503.

7. Alani S, Soyfer V, Strauss N, Schifter D, Corn BW. Limited advantages of intensity-modulated radiotherapy over 3D conformal radiation therapy in the adjuvant management of gastric cancer. Int J Radiat Oncol Biol Phys. 2009;74(2):562-6.

8. Ma H, Han J, Zhang T, Ke Y. Comparison of dosiology between three dimensional conformal and intensity-modulated radiotherapies (5 and 7 fields) in gastric cancer post-surgery. J Huazhong Univ Sci Technolog Med Sci. 2013;33(5):759-64.

9. Otto K. Volumetric modulated arc therapy: IMRT in a single gantry arc. Med Phys. 2008;35(1):310-7.

10. Popescu CC, Olivotto IA, Beckham WA, Ansbacher W, Zavgorodni S, Shaffer $\mathrm{R}$, et al. Volumetric modulated arc therapy improves dosimetry and reduces treatment time compared to conventional intensity-modulated radiotherapy for locoregional radiotherapy of left-sided breast cancer and internal mammary nodes. Int J Radiat Oncol Biol Phys. 2010;76(1):287-95.

11. Palma D, Vollans E, James $K$, Nakano S, Moiseenko V, Shaffer $R$, et al. Volumetric modulated arc therapy for delivery of prostate radiotherapy: comparison with intensity-modulated radiotherapy and three-dimensional conformal radiotherapy. Int J Radiat Oncol Biol Phys. 2008;72(4):996-1001.

12. Bree I, van Hinsberg MG, van Veelen LR. High-dose radiotherapy in inoperable nonsmall cell lung cancer: comparison of volumetric modulated arc therapy, dynamic IMRT and 3D conformal radiotherapy. Med Dosim. 2012;37(4):353-7.

13. Wang X, Li G, Zhang Y, Bai S, Xu F, Wei Y, et al. Single-arc volumetricmodulated arc therapy (sVMAT) as adjuvant treatment for gastric cancer: Dosimetric comparisons with three-dimensional conformal radiotherapy (3D-CRT) and intensity-modulated radiotherapy (IMRT). Med Dosim. 2013;38(4):395-400.

14. Egner JR. AJCC cancer staging manual. JAMA. 2010;304(15):1726-7.
15. Chavaudra J, Bridier A. Definition of volumes in external radiotherapy: ICRU reports 50 and 62. Cancer Radiother. 2001;5(5):472-8.

16. Matzinger $O$, Gerber $E$, Bernstein Z, Maingon P, Haustermans K, Bosset $J F$, et al. EORTC-ROG expert opinion: radiotherapy volume and treatment guidelines for neoadjuvant radiation of adenocarcinomas of the gastroesophageal junction and the stomach. Radiother Oncol. 2009;92(2):164-75.

17. Dawson LA, Kavanagh BD, Paulino AC, Das SK, Miften M, Li XA, et al. Radiation-associated kidney injury. Int J Radiat Oncol Biol Phys. 2010;76(3 Suppl):S108-15.

18. Nicolini G, Ghosh-Laskar S, Shrivastava SK, Banerjee S, Chaudhary S, Agarwal $J P$, et al. Volumetric modulation arc radiotherapy with flattening filter-free beams compared with static gantry IMRT and 3D conformal radiotherapy for advanced esophageal cancer: a feasibility study. Int J Radiat Oncol Biol Phys. 2012;84(2):553-60.

19. Torrens M, Chung C, Chung HT, Hanssens P, Jaffray D, Kemeny A, et al. Standardization of terminology in stereotactic radiosurgery: Report from the Standardization Committee of the International Leksell Gamma Knife Society: special topic. J Neurosurg. 2014;121(Suppl):2-15.

20. Knoos T, Kristensen I, Nilsson P. Volumetric and dosimetric evaluation of radiation treatment plans: radiation conformity index. Int J Radiat Oncol Biol Phys. 1998;42(5):1169-76.

21. Wu WW, Kwong DL, Sham JS. Target dose conformity in 3-dimensional conformal radiotherapy and intensity modulated radiotherapy. Radiother Oncol. 2004;71(2):201-6.

22. Kataria T, Rawat S, Sinha SN, Garg C, Bhalla NK, Negi PS. Dose reduction to normal tissues as compared to the gross tumor by using intensity modulated radiotherapy in thoracic malignancies. Radiat Oncol. 2006;1:31.

23. Van Benthuysen L, Hales L, Podgorsak MB. Volumetric modulated arc therapy vs. IMRT for the treatment of distal esophageal cancer. Med Dosim. 2011;36(4):404-9.

24. Yin Y, Chen J, Xing L, Dong X, Liu T, Lu J, et al. Applications of IMAT in cervical esophageal cancer radiotherapy: a comparison with fixed-field IMRT in dosimetry and implementation. J Appl Clin Med Phys. 2011;12(2):3343.

25. Kuijper IT, Dahele M, Senan S, Verbakel WF. Volumetric modulated arc therapy versus conventional intensity modulated radiation therapy for stereotactic spine radiotherapy: a planning study and early clinical data. Radiother Oncol. 2010;94(2):224-8.

26. Pan CC, Kavanagh BD, Dawson LA, Li XA, Das SK, Miften M, et al. Radiation-associated liver injury. Int J Radiat Oncol Biol Phys. 2010;76(3 Suppl):S94-100.

27. Dawson LA. The evolving role of radiation therapy in hepatocellular carcinoma. Cancer Radiother. 2008;12(2):96-101.

28. Kuo YC, Chiu YM, Shih WP, Yu HW, Chen CW, Wong PF, et al. Volumetric intensity-modulated Arc (RapidArc) therapy for primary hepatocellular carcinoma: comparison with intensity-modulated radiotherapy and 3-D conformal radiotherapy. Radiat Oncol. 2011;6:76.

29. Jansen EP, Saunders MP, Boot H, Oppedijk V, Dubbelman R, Porritt B, et al Prospective study on late renal toxicity following postoperative chemoradiotherapy in gastric cancer. Int J Radiat Oncol Biol Phys. 2007;67(3):781-5.

30. Minn AY, Hsu A, La T, Kunz P, Fisher GA, Ford JM, et al. Comparison of intensity-modulated radiotherapy and 3-dimensional conformal radiotherapy as adjuvant therapy for gastric cancer. Cancer. 2010;116(16):3943-52.

31. Kirkpatrick JP, van der Kogel AJ, Schultheiss TE. Radiation dose-volume effects in the spinal cord. Int J Radiat Oncol Biol Phys. 2010;76(3 Suppl):S42-9. 